\title{
Microemulsions: A Landau-Ginzburg Theory
}

\author{
Kan Chen, ${ }^{(1)}$ C. Jayaprakash, Rahul Pandit, ${ }^{(3)}$ and Wolfgang Wenzel (2) \\ '"Physics Department, Brookhaten National Laboratory, Upton, New York I 1973 \\ "'Department of Physics, The Ohio State University, Columbus, Ohio 43210 \\ ${ }^{(3)}$ Department of Physics, Indian Institute of Science, Bangalore 560012, India
}

\begin{abstract}
We present a Landau-Ginzburg theory for oil-water-surfactant mixtures with a scalar order parameter $\psi$ for the oil-water subsystem and a vector $\tau$ for the surfactant part. A physical interpretation of the parameters in terms of single-membrane energetics is given. A detailed density-functional calculation yields three-phase coexistence between oil-rich, water-rich, and microemulsion phases, and modulated phases including lamellar, columnar, and cubic phases. The computed structure factor for water in the microemulsion phase shows a variety of trends in qualitative accord with experiments.
\end{abstract}

PACS numbers: $82.70 . \mathrm{Kj}, 64.70 .-\mathrm{p}$

Oil-water-surfactant mixtures exhibit ${ }^{1-5}$ a variety of phases and phase equilibria. Surfactant molecules reduce the bare oil-water interfacial tension and hence favor the formation of bulk phases with a large density of microscopic oil-water interfaces. Phase equilibria in such systems can be quite complex; ${ }^{3}$ here we concentrate on some of the experimentally observed phenomena.

Phenomenological ${ }^{6-10}$ theories have been successful in accounting for some of these phases, their equilibria, and the correlation functions found therein. In this paper we present a simple Landau-Ginzburg theory for oil-watersurfactant mixtures with many appealing features. We discuss the results of a detailed, physically motivated density-wave-theory analysis for the free-energy functional obtained within a one-loop expansion. The phase diagram includes three-phase coexistence between oilrich, water-rich, and microemulsion phases, and a variety of modulated phases including lamellar, hexago- nal, and cubic phases. The structure factor of the disordered phase of the model is similar to that found in microemulsions, permitting us to identify this regime with the microemulsion phase, in agreement with the prediction of a lattice model we had developed earlier." The dependence of various features of the structure factor on different parameters shows trends similar to experimental systems. We provide a single-membrane interpretation of some of the coupling constants in terms of curvature energies and use this to correlate different structure factors with different microgeometries

Our Landau-Ginzburg theory is based on two order parameters, $\psi$ and $T: \psi$ is large and positive (negative) in the water-rich (oil-rich) phase and $\tau$ characterizes the surfactant part $[|\boldsymbol{\tau}|$ is large in surfactant-rich regions and $\tau(\boldsymbol{r})$ points in the direction of the heads of surfactant molecules at $\mathbf{r}$ ]. We display the free-energy functional that is oil-water symmetric: ${ }^{12}$

$$
\begin{gathered}
\mathcal{F}=\int d \mathbf{r}\left\{\frac{1}{2} c_{1}(\nabla \psi)^{2}+\frac{1}{2} c_{2}\left(\nabla^{2} \psi\right)^{2}+\frac{1}{2} c_{3}\left(\nabla^{2} \psi\right)^{3}+a_{1} \psi+\frac{1}{2} a_{2} \psi^{2}+a_{4} \psi^{4}+a_{6} \psi^{6}\right. \\
\left.+\frac{1}{2} r_{0}|\boldsymbol{\tau}|^{2}+\frac{1}{2} K_{1}(\nabla \cdot \tau)^{2}+r_{2} \psi^{2}|\boldsymbol{\tau}|^{2}-\boldsymbol{\tau} \cdot \nabla\left(J_{1} \psi+J_{2} \nabla^{2} \psi\right)\right\}
\end{gathered}
$$

The parameter $c_{1}(>0)$ is a measure of the bare oilwater interfacial tension. Since the surfactant molecules can change the sign of $c_{1}$, we include higher-order gradients for stability. $a_{1}$ is proportional to the difference between the chemical potentials of water and oil. The parameters $r_{0}$ and $K_{1}$ characterize the surfactant and are chosen positive so that the surfactant subsystem does not order on its own. The last three terms describe interactions between $\tau$ and $\psi, r_{2}$ is a measure of the miscibility of the surfactant in water and oil. The last two terms account for the amphiphilicity of the surfactant molecules: $J_{1}>0$ favors surfactant molecules residing at oil-water interfaces. The interpretation of the $J_{2}$ term is discussed below; a continuum approximation to a microscopic model would naturally contain such higher-derivative terms. Only terms quadratic in $\tau$ are considered because we will not address the ordering of surfactant molecules in the absence of oil and/or water in this paper.
An intuitive interpretation of the terms proportional to $c_{1}, c_{2}, J_{1}$, and $J_{2}$ can be obtained in the language appropriate to interfaces, ${ }^{13}$ allowing us to make connections between our bulk theory and the interface approach developed by others. ${ }^{13-15}$ Assume that the interface thickness is of order $l$, the length of a surfactant molecule, and that the surfactant molecules are aligned exactly along the local interface normal $\hat{\mathbf{n}}$, i.e., $\tau=\tau_{0} \hat{\mathbf{n}}$, with $\tau_{0}$ the surfactant density at the interface. If $+\psi_{0}$ $\left(-\psi_{0}\right)$ is the bulk value of $\psi$ in water (oil), then the terms proportional to $J_{1}$ and $c_{1}$ clearly represent surface-tension effects. Using elementary differential geometry one can argue that the $c_{2}$ term yields $\left(2 c_{2} \psi_{0}^{2} / l\right)$ $\times \int d s H^{2}$ and the $J_{2}$ term, $J_{2} \tau_{0} \psi_{0} \int d s\left(4 H^{2}-2 K\right)$, where $H$ is the local mean curvature, and $K$ the local Gaussian curvature. Thus by tuning $c_{1}, c_{2}, J_{1}$, and $J_{2}$ the effective surface tension and the curvatures of the internal micro. 
scopic interfaces that constitute the microemulsion phase can be varied. If the effective surface tension is negligible, then these interfaces are like the membranes envisioned by de Gennes and Taupin? and the term proportional to $c_{2}$ incorporates the physics pointed out by them for a single interface: When $J_{1}$ reduces the effective coefficient of the $(\nabla \psi)^{2}$ term to zero, the $c_{2} \int q^{2} n_{q}^{2} d s$ term leads to a logarithmic divergence for the appropri- ate correlation function.' A term which favors spontaneous curvature can be incorporated easily, e.g., by adding the term $\int d^{3} r\left(\nabla^{2} \psi\right) \tau \cdot \nabla \psi \propto \int H d s$ to Eq. (1). The sign of this term can be chosen to agree with Bancroft's rule: The best solvent of the amphiphile is "outside."

For nonzero $r_{2}$ we have calculated the one-loop correction to the free-energy functional by integrating out the surfactant degrees of freedom. Neglecting the $q$-dependent corrections to the $\psi^{4}$ term we obtain

$$
\begin{aligned}
\mathcal{F}_{\mathrm{eff}}^{(2)}= & \int \frac{d^{3} q}{(2 \pi)^{3}}\left[\frac{a_{2}^{\mathrm{er}}}{2}+\left[c_{1}-\frac{\left(J_{1}-J_{2} q^{2}\right)^{2}}{r_{0}+K_{1} q^{2}}\right] \frac{q^{2}}{2}+\frac{c_{2} q^{4}}{2}+\frac{c_{3} q^{6}}{2}\right] \psi(\mathbf{q}) \psi(-\mathbf{q}) \\
& +\int d^{3} r\left\{a_{1} \psi(\mathbf{r})+\left[a_{4}-3 r_{2}^{2} I_{2}(0)\right] \psi^{4}(\mathbf{r})+a_{6} \psi^{6}(\mathbf{r})\right\}
\end{aligned}
$$

where

$$
a_{2}^{\mathrm{eff}}=a_{2}+\frac{1}{2 \pi^{2}}\left\{\frac{\Lambda}{K_{1}}-\frac{\sqrt{r_{0}}}{K_{1}^{3 / 2}} \tan ^{-1}\left(\frac{\Lambda \sqrt{K_{1}}}{\sqrt{r_{0}}}\right)\right\},
$$

$\mathbf{A}$ is the upper cutoff on the momentum, and

$$
I_{2}(0)=\frac{1}{4 \pi^{2} K_{1}^{2}}\left(\frac{K_{1}}{r_{0}}\right)^{1 / 2}\left(\tan ^{-1}(x)-\frac{x}{1+x^{2}}\right),
$$

with $\mathbf{x}=\Lambda\left(K_{1} / r_{0}\right)^{1 / 2}$. The complete expression including odd terms will be reported elsewhere.

The form of the free energy in Eq. (2) has been used for the calculations reported in this paper. We identify the coefficient of the quadratic term as $\frac{1}{2}\left[S^{-1}(q)\right]$ and relabel the coefficient of the $\psi^{4}$ term $u$ and that of the $\psi^{6}$ term $v$ for convenience. We have investigated the stabilities of disordered and uniform oil-rich and water-rich

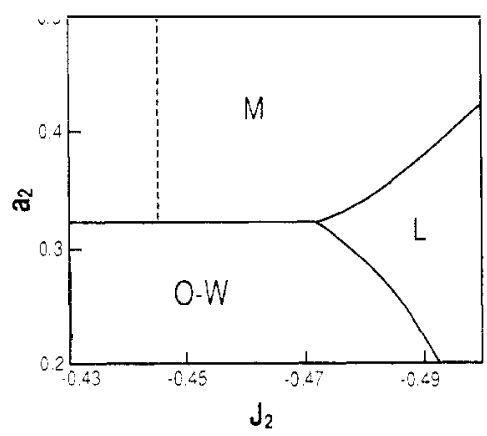

FIG. 1. The phase diagram in the $a_{2}-J_{2}$ plane. Parameters for this phase diagram are $a_{1}=0, u=-0.8, v=1.0, J_{1}=0.39$, $r_{0}=K_{1}-0.16, c_{1}=c_{2}=1$, and $c_{3}=\frac{1}{3}$. Microemulsion and lamellar phases are denoted, respectively, by $M$ and $L$. The region marked $O-W$ shows where the oil-rich and water-rich phases coexist (note $a_{1}=0$ ). The solid lines indicate first-order phase boundaries which meet at a triple point. The dashed line denotes the disorder line, to the left of which $S(q)$ has only one peak at $q=0$ and to the right of which there is a peak at nonzero wave vector $\boldsymbol{Q}$ (see Fig. 2). The first-order transitions become continuous via tricritical points if $u$ becomes positive. phases relative to periodically modulated phases-onedimensional (lamellar), two-dimensional (hexagonal), and three-dimensional (various cubic)-by using a density-wave theory of freezing. ${ }^{16}$

The phase diagram that we have obtained using a density-wave theory (see below) is shown in Fig. 1 (for $u=-0.8, v=1.0, r_{0}=0.16$, and $J_{1}=0.39$ ). There are four phases: microemulsion, oil-rich, water-rich, and lamellar. For small negative values of $J_{2}$ and at lower temperatures (or effective $a_{2}$ ) the oil-rich and water-rich phases coexist; as $J_{2}$ becomes more negative, leading to a finite-q peak in the structure factor, they give way to the lamellar phase. At higher temperatures, a microemul. sion phase is obtained. All phase boundaries are first order which become continuous for positive $u$ with a tricritical point in between. Note the line of three-phase coexistence along which oil, water, and microemulsion phases coexist. The interfacial tensions between these coexisting phases is low for small $u$, i.e., in the vicinity of a tricritical point. This mechanism for low interfacial tensions is common to many lattice models of $\mathrm{mi}$ croemulsions. The dashed line in Fig. 1 is the disorder line (not a phase boundary). To the left of it, $S(q)$ has a

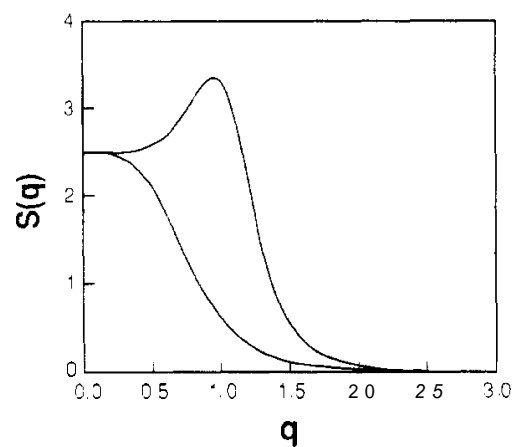

FIG. 2. The structure factor $S(q)$ in the microemulsion phase of Fig. 1. The lower curve $\left(a_{2}-0.4, J_{2}=-0.2\right)$ is obtained in the region to the left of the disorder line of Fig. 1; the upper curve $\left(a_{2}=0.4, J_{?}=-0.492\right)$, to the right of it and close to the microemulsion-lamellar phase boundary whose proximity is signaled by the strong peak. 
peak only at zero (see lower curve in Fig. 2); to the right of it, $S(q)$ has a peak at nonzero $q$ with the extremum at $q=0$ either lower or higher than the peak at nonzero $q$. The upper curve in Fig. 2 shows a structure factor with a prominent peak at nonzero $q$. While we cannot control the density variables explicitly, it is easy to argue that decreasing $r_{0}$ leads to an increase in the surfactant concentration; when $r_{0}$ is decreased we find that the lamellar phase is favored, in agreement with experimental trends.'

The density-wave-theory calculations were done by retaining the order parameters $\psi_{0}=\psi(q=0)$ and $\psi(Q)$, where $Q=|\mathbf{G}|$. The G's belong to the first set of reciprocal-lattice vectors for the given periodic structure and $S(q)$ has a maximum at Q. A global maximum of $S(q)$ at Q favors ordering into a periodically modulated phase at $q=Q$, whereas a maximum at $q=0$ leads to a uniform (oil- or water-rich) phase. For reasonable values of $r_{2}$, the one-loop corrections lead to a negative value of $u$. This results in three-phase coexistence between the microemulsion, oil-rich, and water-rich phases; with increasing $J_{1}$ and $J_{2}$, one expects to find coexistence between microemulsion and spatially modulated phases. A somewhat lengthy calculation leads to the following effective free energy within our density-wave theory:

$$
\begin{aligned}
\mathcal{F}_{\mathrm{eff}}= & \frac{1}{2} \psi_{0}\left\{S^{-1}(0) \psi_{0}+\frac{1}{4} N \psi_{Q}\left\{S^{-1}(Q)\right\} \psi_{Q}+u \psi_{0}^{4}+3 N u \psi_{0}^{2} \psi_{Q}^{2}+3 u\left[\frac{1}{8} N(2 N-1)+n_{\square}\right] \psi_{Q}^{4}+\imath \psi_{0}^{6}\right. \\
& +\frac{15}{2} N v \psi_{0}^{4} \psi_{Q}^{2}+45\left[\frac{1}{8} N(2 N-1)+n_{\square}\right] \cdot \psi_{0}^{2} \psi_{Q}^{4} \\
& +\frac{5}{16} \iota \cdot\left[N+9 N(N-1)+6 N(N-1)(N-2)+9 n_{\Delta}+144 n_{\square}+72 n_{\square}(N-4)+18 n_{\Delta} n_{\square}\right] \psi_{Q}^{6} .
\end{aligned}
$$

In the preceding, $N$ denotes the number of reciprocallattice vectors and $n_{\square}$ and $n_{\Delta}$ the number of distinct noncoplanar quadrilaterals and triangles that can be formed with the reciprocal-lattice vectors. We have minimized the free energy numerically among the various competing phases. An aside on the calculation: When $S(q)$ has only one maximum at $q=0$, then we use $S(Q)=S\left(Q^{*}\right)$, where $Q^{*}$ is the value of $q$ at which a maximum appears first as some parameter, say, $J_{2}$, is varied. This simple idea provides a natural way of suppressing modulated phases when there is no peak in $S(q)$ at finite $q$ without introducing any unphysical phase boundaries and without resorting to extensive numerics. We have checked the accessibility of the parameters used by solving numerically the coupled Dyson equations for the self-energy to one-loop order. The details of these calculations will be reported elsewhere. ${ }^{12}$

We have also obtained the phase diagram in the presence of oil-water asymmetric terms. ${ }^{12}$ The inclusion of the cubic term, i.e., the $g \psi^{3}$ term, makes the hexagonal (columnar) and bcc phases stable in different parts of parameter space; thus a variety of phases that occur experimentally are obtained within our density-functional theory.

Experiments on microemulsions yield structure factors with one or two maxima. In our model, neglecting the asymmetry between oil and water, we have $S_{W W}=S_{O O}$ $=-$ Sow, where $S_{w w}, S_{O o}$, and Sow are the water. water, oil-oil, and oil-water partial structure factors, respectively. We now correlate our predictions for $S_{w}$ from our Landau theory and the microstructures they imply with the experimental data.

Consider first the structure factor $S_{W W}(\mathbf{q})$ in the disordered phase for $J_{2}=0$ when there is no free-energy cost for configurations with large Gaussian curvature; the structure factor can have only one maximum either at $q=0$ or at $q=Q \neq 0$. The coefficient of the $q^{2}$ term in $[S(q)]^{-1}$ can become negative, thus favoring the forma- tion of a large density of oil-water interfaces. The value of $Q$, where $S(q)$ has a maximum, depends on $c_{2}$. A peak at $q \neq 0$ in the disordered phase favors the formation of periodically modulated phases as emphasized earlier (see Fig. 2). Thus when the disordered phase coexists with uniform phases (oil and water rich), then $S(q)$ has a peak at $q=0$ and there may or may not be a smaller peak at $q=Q$. The positive definiteness of $c_{2}$ implies that the local curvature should be small. Therefore, when the fraction of oil (or water) is small, a mixed droplet phase occurs, whereas for nearly equal amounts of oil and water a bicontinuous phase with low mean curvature is favored.

For $J_{2}>0$, the energetics favor spherical droplets for given values of the mean curvature. In this case, depending on the value of $J_{1} / \sqrt{r_{0}}$, one obtains a single peak which can occur away from $q=0$ or two peaks. The width of the peak is approximately half the peak position. This situation is similar to the experiments on sodium di-2-ethylhexylsulfosuccinate (AOT), water, and decane. ${ }^{5}$ We have checked that as $r_{0}$ decreases (i.e., as the surfactant concentration increases) the peaks broaden and move outward, reflecting the experimental trend (the increase in $a_{2}^{\text {eff }}$ when $r_{0}$ decreases due to oneloop effects is important for this").

In the case $J_{2}<0$ we can also arrange to have two peaks in the structure factor in the microemulsion phase, one at $q=0$ and the other at $q=Q \neq 0$. (This is the case for parts of the phase diagram shown in Fig. 1.) The width of the peak at $\mathrm{Q}$ is of the same order as $\mathrm{Q}$ itself and is relatively insensitive to the parameter values. The peaks in this case are slightly narrower than in the case when $J_{2}=0$. Our conclusion that two peaks in the structure factor occur when the Gaussian curvature is large and negative is consistent with previous results. ${ }^{18}$ Assuming a cutoff $\mathbf{A}$ of the order of $2 \pi / l$, where $l$ is the surfactant length, we find that the peak occurs at length 
scales that correspond to 15-20 times longer than 1. For moderate values of $J_{2}$, as $r_{0}$ is decreased (or as the surfactant concentration is increased), the second peak moves outward and becomes sharper,' Large and negative values of $J_{2}$ favor configurations with internal interfaces that have large negative Gaussian curvature and low mean curvature (since $c_{2}>0$ ) leading to a phase where the interface has many handles. This phase would correspond to the "plumber's-nightmare" phase, ${ }^{15.19}$ without long-range order. This can undergo a transition into an ordered structure: We have checked by explicit computation in the presence of oil-water asymmetric terms that, as the value of $J_{2}$ is tuned from negative to positive, the morphology evolves from a cubic phase (to be associated with a plumber's-nightmare phase with long-range bcc order), through a first-order transition into a tense bicontinuous structure (or a random. isotropic microemulsion phase depending on the parameters), and eventually into a cubic structure that can be associated with a droplet crystal. Such a scenario is present in a schematic phase diagram of Ref. 15. Note that the inclusion of Gaussian curvature which allows effectively for the formation of passages and fusion of membranes is crucial.

We thank C. Ebner, $Y$. He, H. R. Krishnamurthy, T. V. Ramakrishnan, and S. Ramaswamy for discussions. This research was supported in part by NSF Grant No. NSF-DMR 8451911 , by the Department of Energy, Office of Basic Energy Sciences (DE-FG0288ER 13916), and by an IBM Pre-doctoral Fellowship (W.W.). K.C. was supported in part by the Division of Materials Science, U.S. DOE Contract No. DE-AC0276CH00016. R.P. thanks the University Grants Commission and the Department of Science and Technology (India) for financial support, and The Ohio State University for hospitality. We acknowledge a grant of Cray time from the Ohio Supercomputer Center.

'Physics of Amphiphiles: Micelles. Vesicles. and Microemulsions, edited by V. Degiorgio and M. Corti (Academic, New York, 1985).

'Physics of Amphiphilic Layers, edited by J. Meunier, D. Langevin, and N. Boccara (Springer-Verlag, Berlin, 1987).

${ }^{3}$ M. Kahlweit and R. Strey, Angew. Chem. Int. Ed. Engl. 24, 654 (1985).

${ }^{4}$ A. DeGeyer and J. Tabony, Chem. Phys. Lett. 113, 83 (1985): J. Tabony, Chem. Phys. Lett. 113, 75 (1985)

${ }^{5}$ M. Kotlarchyk, Physica (Amsterdam) 136B, 274 (1986); L. Auvray, J. P. Cotton, R. Ober, and C. Taupin, Physica (Amsterdam) 136B, 281 (1986).

${ }^{6}$ E. W. Kaler, K. E. Bennet, H. T. Davis, and L. E. Scriven, J. Chern. Phys. 79, 5673 (1983)

'P. G. de Gennes and C. Taupin, J. Phys. Chem. 86, 2294 (1982)

${ }^{8}$ B. Widom, Langmuir 3, 12 (1987).

${ }^{9}$ S. T. Milner, S. A. Safran, D. Andelman, M. E. Cates, and D. Roux, J. Phys. (Paris) 49, 1065 (1988).

${ }^{10}$ L. Golubovic and T. Lubensky, Phys. Rev. A 41, 4343 (1990), and references therein.

${ }^{11}$ K. Chen, C. Ebner, C. Jayaprakash, and R. Pandit, J. Phys. C 20, L361 (1987); Phys. Rev. B 38, 6240 (1988).

${ }^{12} \mathrm{~K}$. Chen, C. Jayaprakash, R. Pandit, and W. Wenzel (to be published).

${ }^{13}$ W. Helfrich, Z. Naturforsch. 28c, 693 (1973).

${ }^{14}$ S. Leibler and D. Andelman, J. Phys. (Paris) 48, 2013 (1987).

${ }^{15}$ D. A. Huse and S. Leibler. J. Phys. (Paris) 49. 605 (1988).

${ }^{16}$ T. V. Ramakrishnan and M. Yussouf, Phys. Rev. B 19, 2775 (1979)

${ }^{17}$ M. Teubner and R. Strey, J. Chem. Phys. 87, 3195 (1987).

${ }^{18}$ N. F. Berk, Phys. Rev. Lett. 58, 2718 (1987).

${ }^{19} \mathrm{~L}$. E. Scriven, in Micellization, Solubilization and $\mathrm{Mi}$ croemulsions, edited by K. L. Mittal (Plenum, New York. 1977). p. 877. 